Article

\title{
FHT: A Novel Approach for Filtering High-Availability Seamless Redundancy (HSR) Traffic
}

\section{Nguyen Xuan Tien and Jong Myung Rhee*}

Department of Information and Communications Engineering, MPEES ARC, Myongji University, Yongin-si, Gyeonggi-do 449-728, Korea; E-Mail: nxtien@gmail.com

* Author to whom correspondence should be addressed; E-Mail: jmr77@mju.ac.kr;

Tel.: +82-10-5227-0419; Fax: +82-31-330-6824.

Academic Editor: Neville Watson

Received: 18 May 2015 / Accepted: 12 June 2015 / Published: 25 June 2015

\begin{abstract}
High-availability seamless redundancy (HSR) is a protocol for Ethernet networks that provides duplicated frames with zero recovery time in the event of any network component's failure. It is suited for applications that demand high availability and a very short time-outs such as substation automation systems (SAS). However, HSR generates excessive unnecessary unicast frames and spreads them throughout connected-ring networks, whether or not the destination node exists in network's rings. This unnecessary redundant traffic causes high bandwidth consumption, resulting in degradation of network performance. In this paper, we introduce a novel approach for filtering and reducing HSR unicast traffic in connected-ring networks, called "filtering HSR traffic" (FHT). The purpose of FHT is to filter HSR unicast traffic and remove circulated traffic for all rings in connected-ring networks. Therefore, FHT significantly reduces network unicast traffic in connected-ring networks. The traffic performance of FHT has been analyzed, evaluated, and compared to that of standard HSR protocol and the port locking (PL) approach. Various simulations were conducted to validate the traffic performance analysis. Analytical and simulation results showed that, for our sample network, FHT reduced network unicast traffic by about $82 \%$ compared with standard HSR and by about $56 \%$ compared with the PL approach, thus freeing up network bandwidth and improving network traffic performance.
\end{abstract}

Keywords: high-availability seamless redundancy (HSR); filtering HSR traffic (FHT); fault-tolerant network; substation automation system (SAS) 


\section{Introduction}

Highly seamless communication with fault tolerance is one of the key requirements for Ethernet-based, mission-critical, real-time systems, such as substation automation system (SAS) networks and other industrial Ethernet networks [1]. A fault-tolerant Ethernet (FTE) system is a common solution to address fault tolerance problems in Ethernet-based systems. Several FTE protocols have been developed and standardized in the International Electrotechnical Commission (IEC) 62439 standard series to provide protocol-independent redundancy in substation automation networks, such as media redundancy protocol [2], cross-network redundancy protocol [3], beacon redundancy protocol [4], parallel redundancy protocol (PRP), and high-availability seamless redundancy (HSR) [5]. Shortest Path Bridging (SPB), specified in the IEEE 802.1aq standard, is the replacement for the older spanning tree protocols [6]. SPB allows all paths to be active with multiple equal cost paths and provides much larger layer 2 topologies. SPB also provides fast connectivity restoration after failure.

However, among those protocols, only two-PRP and HSR - are suitable for seamless communications. Both HSR and PRP are based upon the principle of providing duplicated frames for separate physical paths with zero recovery time [5]. Unlike PRP, which requires dual redundant independent networks, HSR can be applied to any topology, in particular single rings and connected rings, while retaining its property of zero recovery time. HSR is a redundancy protocol for Ethernet networks that is standardized by the International Electrotechnical Commission under IEC 62439-3 Clause 5. HSR provides duplicated frame copies for each frame sent. In other words, the HSR protocol provides two frame copies for the destination node, one from each side. In the fault-free state of the network, the destination node receives two identical frames, passes the first frame to its upper layers, and discards the duplicate. In the case of a link or node fault or malfunction, only one frame is lost. The application on the destination node operates with the remaining frame undisturbed. Therefore, even in the case of a node or link failure, there is no communication interrupt in the network. This feature of the HSR protocol makes it very useful for time-critical and mission-critical systems, such as SAS. Other HSR principles are described and discussed in a study by Kirrmann and Kleineberg [7].

The main problem with HSR is unnecessary traffic due to duplicated frames that are generated and forwarded inside the network. This disadvantage degrades network performance and may cause congestion and delay. Several approaches have been proposed to reduce unnecessary traffic in HSR networks. Nsaif and Rhee [8] proposed two approaches to improve network traffic performance by reducing the unnecessary traffic in HSR networks. The first approach, called quick removing (QR), is suited for ring or connected-ring topologies with multicast or broadcast traffic. QR removes the redundant frame copies from the network when all nodes have received one copy of the sent frame and begin to receive a redundant copy. This approach results in a unicast traffic reduction of $37.5 \%$ compared with the standard HSR protocol. The second approach is the virtual ring (VRing), which involves dividing any closed-loop HSR network into several VRings. This approach reduced network unicast traffic by about 48\%-68\% compared with the standard HSR protocol. Later, Nsaif and Rhee [1] also proposed a dual virtual paths (DVP) algorithm, which automatically establishes VRings between each pair of terminal nodes to send unicast traffic only, instead of duplicating and forwarding copies at random in all directions. The DVP algorithm results in a reduction in unicast traffic of about $70 \%$ compared with the standard HSR protocol. Tien, Nsaif and Rhee [9] proposed an optimal dual paths (ODP) 
approach that establishes optimal dual paths for each connection pair in an HSR network based on the network's topology. The ODP approach results in a unicast traffic reduction of $73 \%$ compared with the standard HSR protocol. Hong and Joe [10] introduced a packet transmission scheme with different periods based on a single-ring topology to reduce the HSR network-traffic load. Abdulsalam and Rhee [11] proposed a unicast traffic reduction method called port locking (PL) for connected-ring networks. The PL approach learns the locations of the source and destination nodes for each connection pair, and then prunes the rings that do not contain the destination node by locking the corresponding ports of the related QuadBox node. However, the PL approach has some limitations due to its learning method-it cannot work with connected-ring networks that have two rings connected by a DANH ring, and it cannot prune unicast traffic for QuadBox rings. Additionally, the PL cannot prune unicast traffic for DANH rings with a link or node failure.

In this paper, we propose a novel approach called "filtering HSR traffic" (FHT) to filter and reduce the HSR unicast traffic in connected-ring networks. FHT provides unicast traffic filtering for both DANH rings and QuadBox rings. Therefore, FHT prunes unicast traffic for all rings of connected-ring networks. Additionally, under the FHT approach, circulated traffic is also removed from the rings. By filtering unicast traffic for all rings and removing circulated traffic from them, FHT significantly reduces HSR unicast traffic in connected-ring networks. The FHT approach also overcomes limitations of the PL approach; FHT works with any connected-ring topologies, prunes HSR unicast traffic for both DANH and QuadBox rings, and filters unicast traffic for DANH rings with a link or node failure.

The rest of this paper is organized as follows: in Section 2, we briefly introduce the standard HSR protocol. Next, in Section 3, we describe the proposed FHT approach. In Section 4, the traffic performance of FHT is analyzed, evaluated, and compared to that of the standard HSR protocol as well as the PL approach. Section 5 describes several simulations and their results to evaluate and validate the traffic performance analysis of FHT. Finally, we provide our conclusions and suggestions for future work in Section 6.

\section{Background}

\subsection{High-Availability Seamless Redundancy (HSR) Nodes}

The HSR protocol defines four node types [5].

- Doubly-attached node for HSR (DANH): A DANH node has two HSR ports sharing the same medium access control (MAC) and Internet protocol (IP) addresses. This allows address management protocols, such as the address resolution protocol (ARP), to operate as usual without modification, which simplifies network engineering. Each DANH node duplicates a non-HSR frame that is generated at the upper layer into two frame copies. It then appends an HSR tag to each copy and sends the two copies over its two DANH ports.

- Single-attached node (SAN): A SAN node is a non-HSR device, such as a commercial printer, server, or laptop, which cannot be directly inserted into HSR networks because it lacks the forwarding capability of an HSR node and does not support the HSR tag. These devices must be attached through a redundancy box (RedBox) node type. 
- RedBox node: A RedBox node has three ports: two are HSR ports and the third is an Ethernet port that any SAN device, such as a PC, can use to engage with the HSR network. The RedBox nodes forward the frames over the HSR network like any other HSR node and act as proxies for all SANs that access them. Therefore, they must keep track of all traffic on behalf of the SANs. The RedBox can also act as a switch for the SANs.

- Quadruple port device (QuadBox): A QuadBox node has four HSR ports that are divided into two pairs connected by an interlink. Each pair shares the same MAC and IP addresses and connects to the same ring. A QuadBox node is used to connect two rings with each other in connected-ring topologies. Figure 1 shows an example of a QuadBox node connecting two rings.

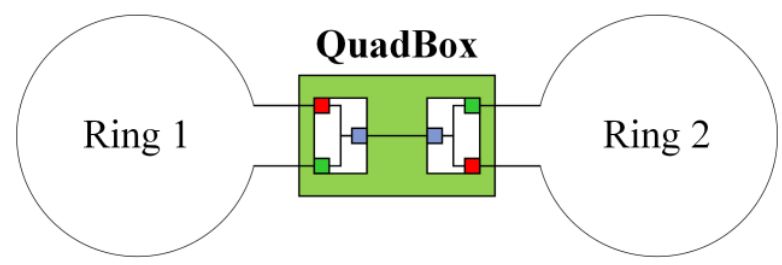

Figure 1. A QuadBox node connecting two rings.

The purpose of FHT is to reduce unnecessary HSR traffic in connected-ring networks. Therefore, DANH and QuadBox nodes are sufficient to handle HSR traffic in this paper.

\subsection{HSR Operation}

The HSR protocol is usually used in single-ring and connected-ring networks. The operation of HSR protocol is described in detail in by IEC 62439-3(5).

\subsubsection{Single-Ring Networks}

A single-ring HSR network consists of DANH nodes, each having two ports, interconnected by full-duplex links, as shown in Figure 2. There is no QuadBox node in single-ring networks. The source node sends two copies of the same frame simultaneously through each port in both directions in the ring. The two frame copies travel in opposite directions. Each node in the HSR network forwards the frame, unless it is the destination of the frame. The destination node of a unicast frame does not forward a frame for which it is the only destination. Frames forwarding in the ring carry the HSR tag inserted by the source node, which contains a sequence number. The doublet (source MAC address, sequence number) uniquely identifies copies of the same frame. In the fault-free case of the network, each destination node receives two identical frames, passes the first frame to its upper layers, and discards the duplicate. In case of a link or a node failure or malfunction, only one frame is lost and the other frame still reaches the destination node.

\subsubsection{Connected-Ring Networks}

In connected-ring networks, HSR rings are connected by QuadBox nodes. A QuadBox node forwards frames over each ring, passing the frames to another ring without changes. Figure 3 shows an example of a connected-ring network that consists of four DANH rings and one QuadBox ring. 


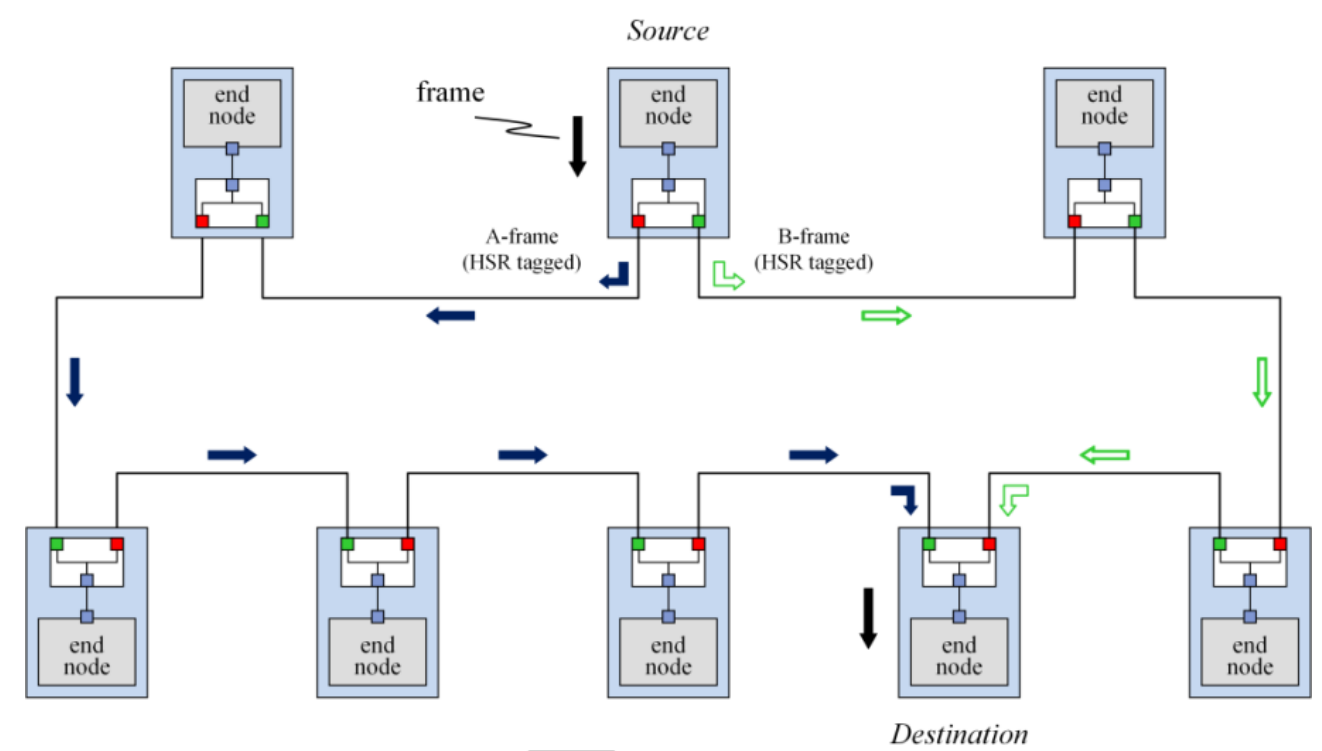

DANH Node

Figure 2. HSR example of single-ring topology for unicast traffic.

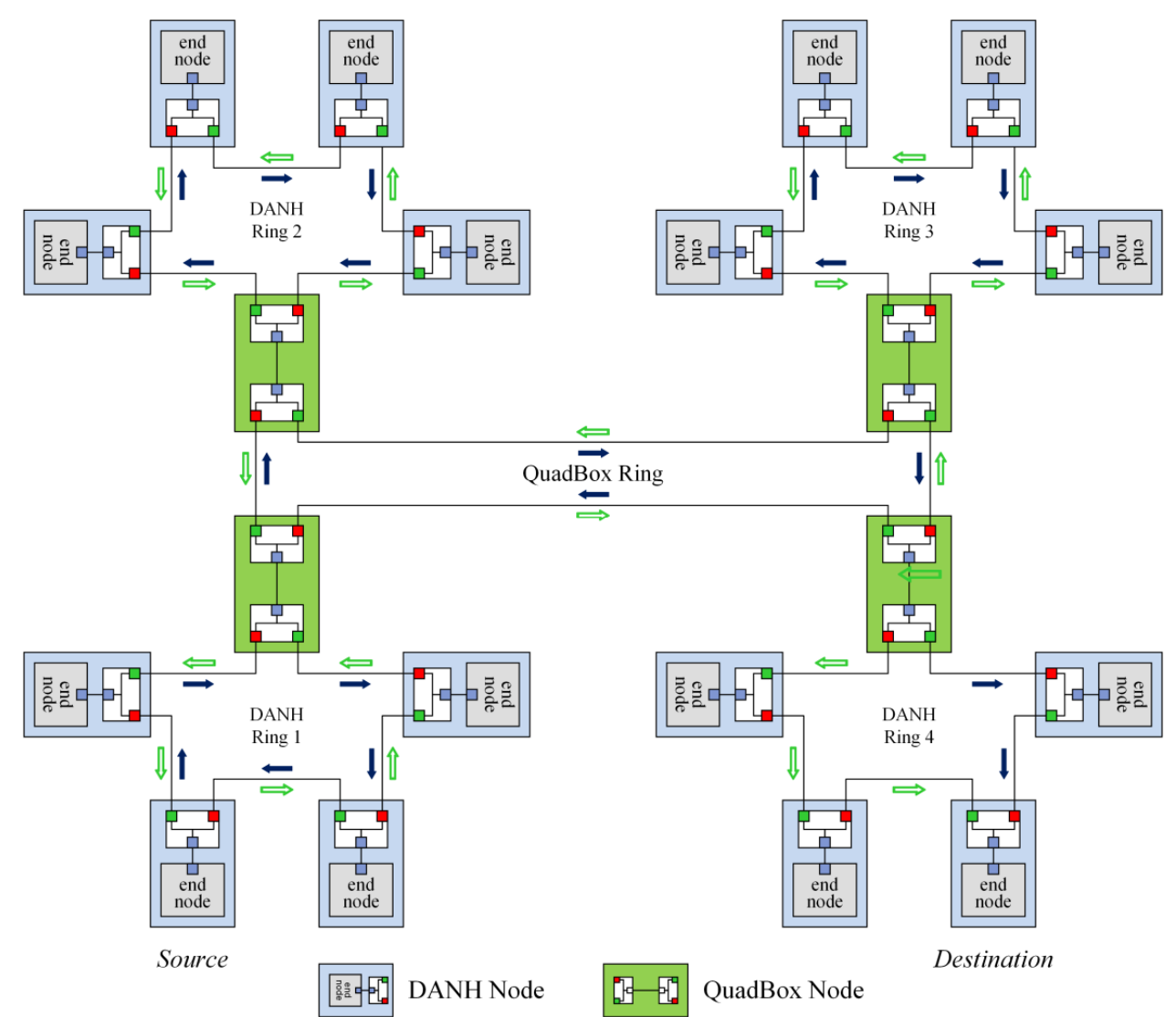

Figure 3. HSR example of connected-ring topology for unicast traffic.

The forwarding rule of the standard HSR protocol is that nodes forward unicast frames from one port to other ports, unless they already sent the same frame in that direction. A node will not forward a frame that it injected into the ring [5].

HSR protocol has no issues with unicast frames inside a single-ring network. However, HSR protocol generates too much unicast traffic in a connected-ring network. In a connected-ring network, 
unicast frames are forwarded and circulated in all rings, except the destination DANH ring, as shown in Figure 3. In other words, there are two frame copies delivered to each link of all rings in the network, one in each direction, except the destination DANH ring. This problem degrades the network performance and may cause network congestion and delay.

\section{The Proposed Filtering HSR Traffic (FHT) Approach}

In this section, we describe the proposed FHT approach for filtering HSR unicast traffic and improving HSR network performance in connected-ring networks.

\subsection{FHT Concepts}

The purpose of FHT is to filter HSR unicast traffic for all rings in HSR connected-ring networks. FHT allows a unicast frame to be forwarded in DANH rings that contain the source node or the destination node of the frame, and in intermediate rings that are used to connect the source and destination DANH rings. The ring terms are defined as follows:

Definition 1 (DANH ring): A DANH ring is a ring in a connected-ring network that consists of DANH nodes and QuadBox nodes. QuadBox nodes in a DANH ring are used to connect the DANH ring to another ring.

Definition 2 (source DANH ring): The source DANH ring of a unicast frame is a DANH ring that contains the source node of the frame.

Definition 3 (destination DANH ring): The destination DANH ring of a unicast frame is a DANH ring that contains the destination node of the frame.

Definition 4 (QuadBox ring): A QuadBox ring is a ring in a connected-ring network that consists of only QuadBox nodes.

Definition 5 (terminal ring): A terminal ring is a DANH ring that is connected to another ring in a connected-ring network.

Definition 6 (intermediate ring): An intermediate ring is a QuadBox or DANH ring that is used to connect two other rings in a connected-ring network.

In this paper, the term "DANH ring" refers to a terminal DANH ring. In the FHT approach, the traffic-filtering function is performed only at QuadBox nodes, not DANH nodes. To filter HSR unicast traffic, QuadBox nodes configure specific port types. FHT defines three port types for QuadBox nodes: access port, trunk port, and gateway port:

Definition 7 (access port): An access port is a QuadBox node port that is directly connected to a DANH ring.

Definition 8 (trunk port): A trunk port is a QuadBox node port that is directly connected to a QuadBox ring.

Definition 9 (gateway port): A gateway port is a QuadBox node port that is directly connected to an intermediate DANH ring.

Based on the port types, FHT defines two types of QuadBox nodes: access QuadBox nodes and trunk QuadBox nodes:

Definition 10 (access QuadBox): An access QuadBox is a QuadBox node that has at least one pair of access ports or gateway ports. An access QuadBox node is connected to at least one DANH ring. 
Definition 11 (trunk QuadBox): A trunk QuadBox node is a QuadBox node that has only trunk ports. A trunk QuadBox node is used to connect two QuadBox rings.

To perform filtering functionality at QuadBox nodes, FHT establishes and maintains two DANH MAC tables: the MAC1 table and the MAC2 table:

Definition 12 (MAC1 table): A MAC1 table is established and maintained at each access QuadBox node and contains MAC addresses of all DANH nodes in DANH rings of the access QuadBox node.

Definition 13 (MAC2 table): A MAC2 table is established and maintained at each trunk QuadBox node and includes MAC addresses from the MAC1 tables of other QuadBox nodes connected to QuadBox rings of the trunk QuadBox node.

The following are some important notes about MAC tables:

- MAC tables contain MAC addresses of DANH nodes, not QuadBox nodes.

- MAC tables are established and maintained at QuadBox nodes, not DANH nodes.

- Each QuadBox node has at most one MAC table, a MAC1 table or a MAC2 table. The MAC1 table is established and maintained at access QuadBox nodes, while the MAC2 table is established and maintained at trunk QuadBox nodes.

To implement the functionality of filtering unicast traffic, FHT defines a set of traffic-filtering rules:

- Filtering rule 1: Filtering inbound traffic for DANH rings. Access QuadBox nodes are used to filter inbound unicast traffic for DANH rings based on their MAC1 table. An access QuadBox node forwards a unicast frame into a DANH ring if and only if the DANH ring contains the destination node of the frame.

- Filtering rule 2: Filtering outbound traffic for QuadBox rings. Trunk QuadBox nodes are used to filter outbound traffic for QuadBox rings based on their MAC2 table. A trunk QuadBox node forwards a unicast frame from its first QuadBox ring to its second QuadBox ring if and only if the first QuadBox ring does not connect to the frame's destination DANH ring.

Additionally, FHT also defines a new traffic-forwarding rule for QuadBox nodes, as follows:

- Forwarding rule: QuadBox nodes forward a unicast frame once, at most. When a QuadBox node receives a unicast frame, it checks if the frame has previously been received and forwarded. If not, it forwards the frame. If so, it discards the frame. This new forwarding rule allows FHT to remove circulated traffic in rings.

Figure $4 \mathrm{a}$ shows the process of filtering inbound unicast traffic for a DANH ring at an access QuadBox node; Figure 4b shows the process of filtering outbound unicast traffic for a QuadBox ring at a trunk QuadBox node.

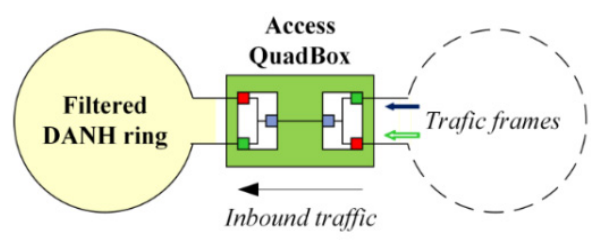

(a) Inbound traffic filtering for a DANH ring

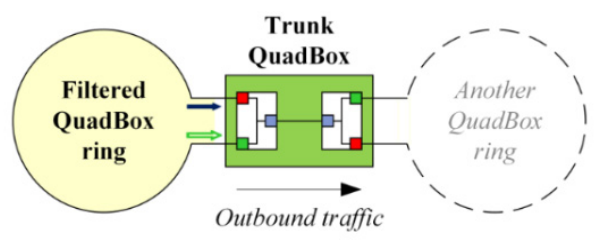

(b) Outbound traffic filtering for a QuadBox ring

Figure 4. The process of filtering unicast traffic. 
Under standard HSR protocol, unicast frames are circulated in rings and duplicated on each link of the rings as shown in Figure 5a; whereas FHT removes the circulation and allows one copy of each frame to be delivered on each link of the rings, as shown in Figure $5 \mathrm{~b}$.

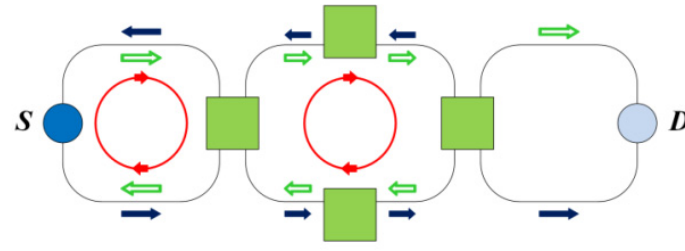

(a) Standard HSR

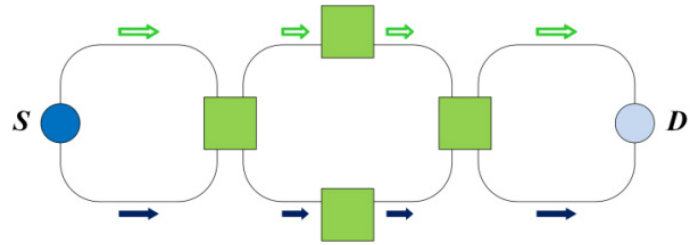

(b) FHT

Figure 5. Forwarding a unicast frame from node $\mathrm{S}$ to node D.

\subsection{FHT Frame Structure}

FHT uses three types of control messages, including Hello messages, ACK (Acknowlegment) messages, and MAC messages, to configure port types and establish and maintain MAC tables for QuadBox nodes. Hello and MAC messages are sent by QuadBox nodes, whereas ACK messages are sent by DANH nodes. Figure 6 shows the frame structure of standard HSR and FHT.

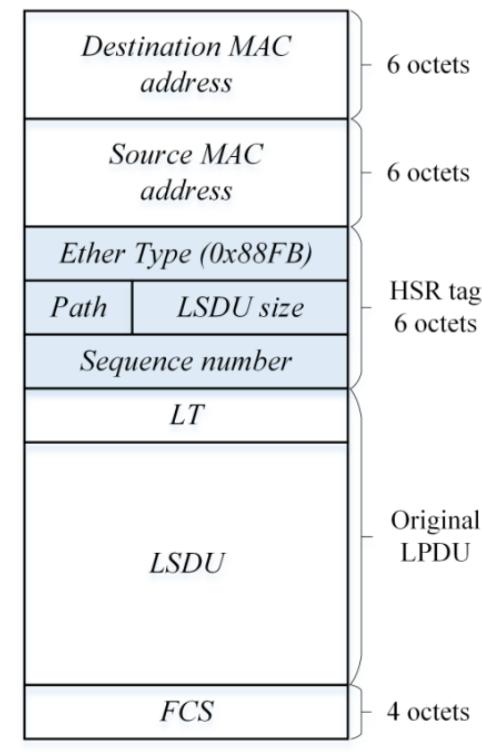

(a) Standard HSR

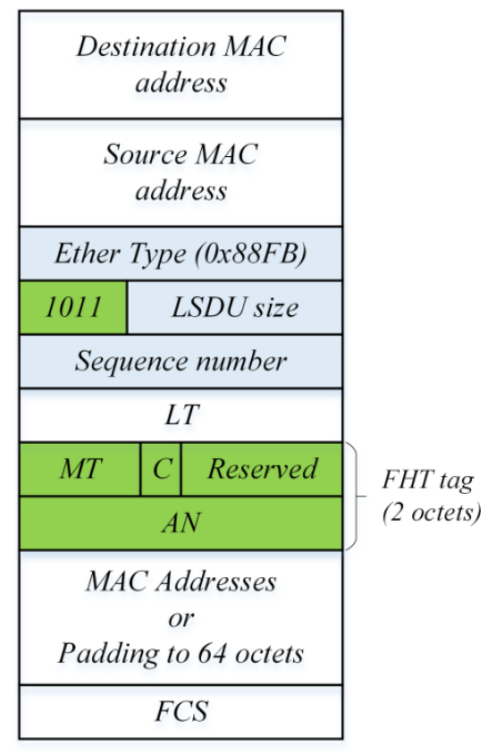

(b) FHT

Figure 6. Frame structure.

Hello and ACK messages are data-less HSR frames with a size of 64 octets. The size of MAC messages depends on the number of MAC addresses included in the message. To distinguish between standard HSR's frames and FHT's control frames, we used one of the reserved codes of the path field located inside the HSR tag. Code 1011 refers to FHT's control frames. Each FHT control frame contains a 2-octet FHT tag that consists of the following control information: 
- $\quad M T$ : 3-bit message type field

000: Hello message

001: ACK message

010: MAC message

011-111: reserved

- $\quad C$ : 1-bit connection field

0 : The sender node of the received frame is not directly connected to the receiver node.

1: The sender node is directly connected to the receiver node.

- $\quad$ Reserved: 4-bit reserved

- $A N$ : 8-bit address number field. This field indicates the number of MAC addresses contained in the message. The field is set to 00000000 for Hello and ACK messages.

\subsection{FHT Operations}

The traffic-filtering functionality of FHT is implemented at QuadBox nodes that are used to connect rings in a connected-ring network. To perform this functionality, FHT first sets port types and then builds DANH MAC tables for the QuadBox nodes.

\subsubsection{Setting Port Type}

Each QuadBox node periodically generates a Hello message with the bit $C$ of 1 and sends over all its ports. When a DANH node receives a Hello message, it performs the following tasks:

- Forwarding the Hello message: The DANH node sets the bit $C$ of the received Hello message to 0 and forwards the updated Hello message to another port.

- $\quad$ Replying by sending an ACK message: The DANH node generates an ACK message with a bit $C$ of 1 and sends it over the port on which the Hello message was received.

When a DANH node receives an ACK message sent by another DANH node, it changes the bit $C$ of the ACK message to 0 and forwards the updated ACK message to another port. QuadBox nodes use Hello and ACK messages to configure port types for all their ports. When a QuadBox node receives a Hello message, it checks the bit $C$ of the Hello message. If the bit $C$ is equal to 1, then the QuadBox received the Hello message from another QuadBox that is directly connected. In response, the QuadBox node sets its received port to the trunk port. If the bit $C$ is equal to 0, the sending QuadBox is not directly connected to the receiving QuadBox. In this case, the QuadBox node sets the received port to the gateway port.

When a QuadBox node receives an ACK message, a DANH node is directly connected to it through the port on which the ACK message was received. The QuadBox node checks whether the received port is set to the gateway port. If so, the QuadBox node keeps the current port type for the received port. If not, it sets the received port to the access port.

After checking the received Hello or ACK messages, the QuadBox nodes discard the message. QuadBox nodes do not forward Hello and ACK messages. The process of setting port types for a QuadBox node is shown in Figure 7. 


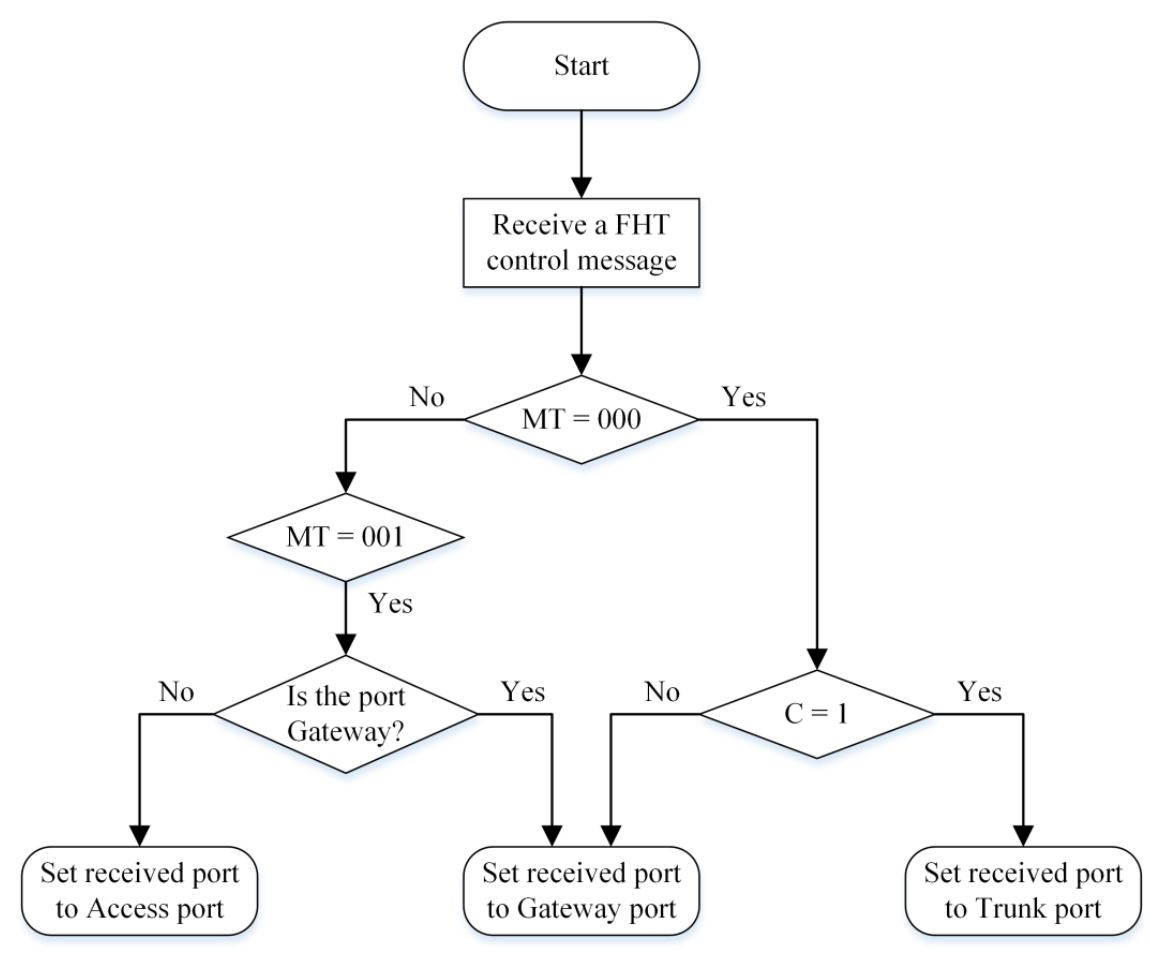

Figure 7. The process of setting port types for a QuadBox node.

\subsubsection{Establishing the MAC1 Table for Access QuadBox Nodes}

The FHT approach filters unicast traffic for DANH rings based on the MAC1 table. An access QuadBox node builds its MAC1 table based on received ACK messages from DANH nodes in its DANH ring. When an access QuadBox node receives an ACK message, it checks if its MAC1 table contains the source MAC address of the ACK message. If not, the QuadBox adds the source MAC address into its MAC1 table. If so, the ACK message is discarded. By learning the MAC addresses of DANH nodes, each access QuadBox knows the MAC addresses of all DANH nodes connected to its corresponding DANH ring. Based on the MAC1 table, an access QuadBox node will not forward an HSR unicast traffic frame into a DANH ring if the DANH ring does not contain the destination node of the frame.

\subsubsection{Establishing the MAC2 Table for Trunk QuadBox Nodes}

The FHT approach filters unicast traffic for QuadBox rings based on the MAC2 table. The process of building a MAC2 table at trunk QuadBox nodes is described as follows: once an access QuadBox node has established its MAC1 table, it sends a MAC message that contains all the MAC addresses of its MAC1 table over trunk ports that are connected to a QuadBox ring. The other QuadBox nodes connected to the QuadBox ring receive the MAC message, update their MAC2 tables if they are trunk QuadBox nodes, and then forward the MAC message to the trunk port connected to the same QuadBox ring if the MAC message is being received for the first time.

Based on the MAC2 table, a trunk QuadBox node will not forward an HSR unicast frame from its first QuadBox ring to its second QuadBox ring if the first QuadBox ring is connected to the destination DANH ring. 


\subsubsection{FHT Operation Principle}

Figure 8 shows the process of filtering a unicast frame under the FHT approach in a connected-ring network with three QuadBox rings used to connect eight DANH rings. We consider the case in which a source node in DANH ring 1 sends a unicast frame to a destination node in DANH ring 5.

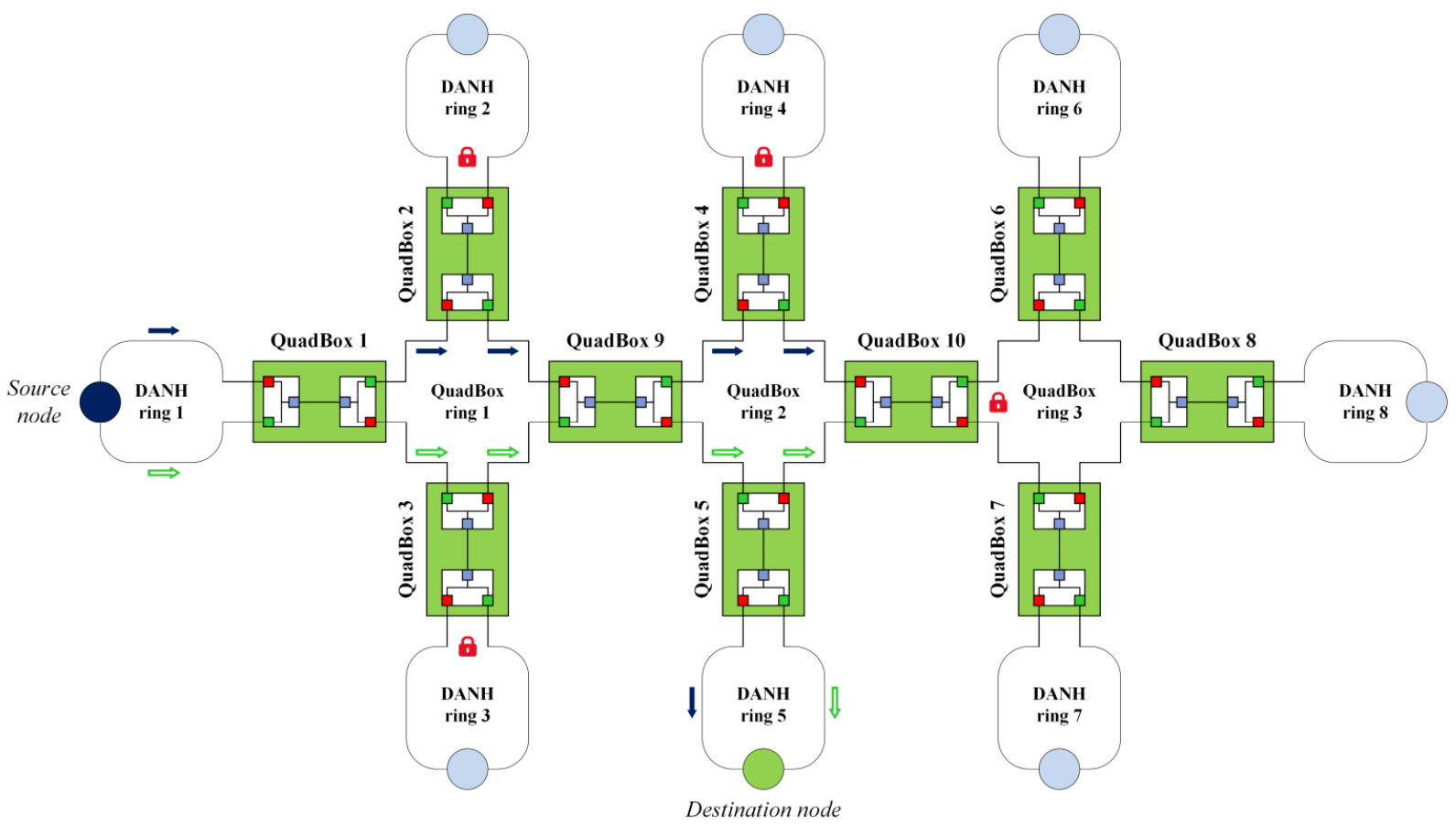

Figure 8. The process of filtering a unicast frame using the Filtering HSR Traffic (FHT) approach.

The source node sends two copies of the frame simultaneously through each port in both directions in DANH ring 1. Access QuadBox 1 passes the frame to QuadBox ring 1. Access QuadBox nodes 2 and 3 look up their MAC1 tables and do not forward the frame to DANH rings 2 and 3, respectively, since these DANH rings do not contain the destination node of the frame. Trunk QuadBox 9 also looks up its MAC2 table and then forwards the frame from QuadBox ring 1 to QuadBox ring 2 because QuadBox ring 1 does not connect to the destination DANH ring of the frame. Similarly, access QuadBox 4 does not forward the frame to DANH ring 4. However, trunk QuadBox node 10 does not forward the frame from QuadBox ring 2 to QuadBox ring 3, since QuadBox ring 2 connects to the destination DANH ring: DANH ring 5. Finally, access QuadBox 5 forwards the frame into DANH ring 5 because DANH ring 5 contains the destination node of the frame. The destination node receives two copies of the frame from both directions of DANH ring 5, passes the first frame to its upper layers, and discards the duplicate.

According to the new forwarding rule for QuadBox nodes, the frame is not circulated and duplicated in intermediate QuadBox rings. In other words, there is only one copy of the frame delivered to each link of the intermediate rings.

Generally, the FHT approach filters unicast traffic for all rings of a connected-ring network so unicast traffic is delivered only in source and destination DANH rings, as well as intermediate rings that connect between source and destination DANH rings. 


\subsubsection{Single Point of Failure Problem}

Although one QuadBox is sufficient to conduct the traffic in a fault-free network, two QuadBox nodes are used to prevent a single point of failure [5]. Therefore, we define some new terms related to paired QuadBox nodes:

Definition 14 (paired QuadBox nodes): Paired QuadBox nodes are two QuadBox nodes that are used to connect two rings to prevent a single point of failure. Figure 9 shows two paired QuadBox nodes used to connect two rings.

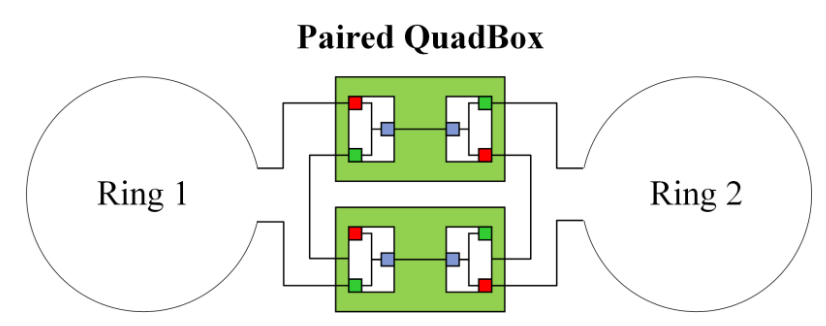

Figure 9. Paired QuadBox nodes connecting two rings.

Definition 15 (paired trunk port): A paired trunk port is a QuadBox node port that is directly connected to its paired QuadBox node.

In FHT, two paired QuadBox nodes functionally operate as a single QuadBox node to connect two rings. Paired QuadBox nodes have the same filtering and forwarding rules as normal QuadBox nodes. Additionally, two paired QuadBox nodes have the same MAC tables as each other. To make two paired QuadBox nodes work as a single QuadBox node, the paired nodes share received messages with each other. When a paired QuadBox node receives messages, including control messages and traffic messages, it always forwards these messages to its paired QuadBox node through the paired trunk port that belongs to the same port pair as the received port. For convenience, we use a single QuadBox node to connect two rings in our traffic performance analysis.

\subsubsection{Compatibility}

FHT nodes can work in FHT mode as well as standard HSR mode. If an HSR network consists of only FHT-capable nodes, the FHT mode will be enabled to reduce network unicast traffic and improve network traffic performance. If FHT-capable nodes connect to non-FHT-capable nodes in an HSR network, the FHT-capable nodes will work in standard HSR mode to prevent malfunction in the network.

\section{Traffic Performance Analysis}

This section describes the traffic performance analysis of FHT compared with standard HSR protocol and the PL approach. To analyze and evaluate traffic performance, network unicast traffic was chosen as a performance metric, because network unicast traffic shows a clear difference between the approaches.

Network unicast traffic in a connected-ring network is defined as the total number of unicast traffic frames that are delivered on links and received by nodes in the network. In this paper, "network traffic" refers to network unicast traffic. When a source node sends a unicast traffic frame to a destination 
node, network traffic in this case is the total number of copies of the frame delivered on links and received by nodes in the network.

We consider a sample connected-ring network consisting of eight DANH rings and three QuadBox rings as shown in Figure 10. Each DANH ring includes four DANH nodes and one QuadBox node, while each QuadBox ring consists of four QuadBox nodes. The source node belongs to DANH ring 1.

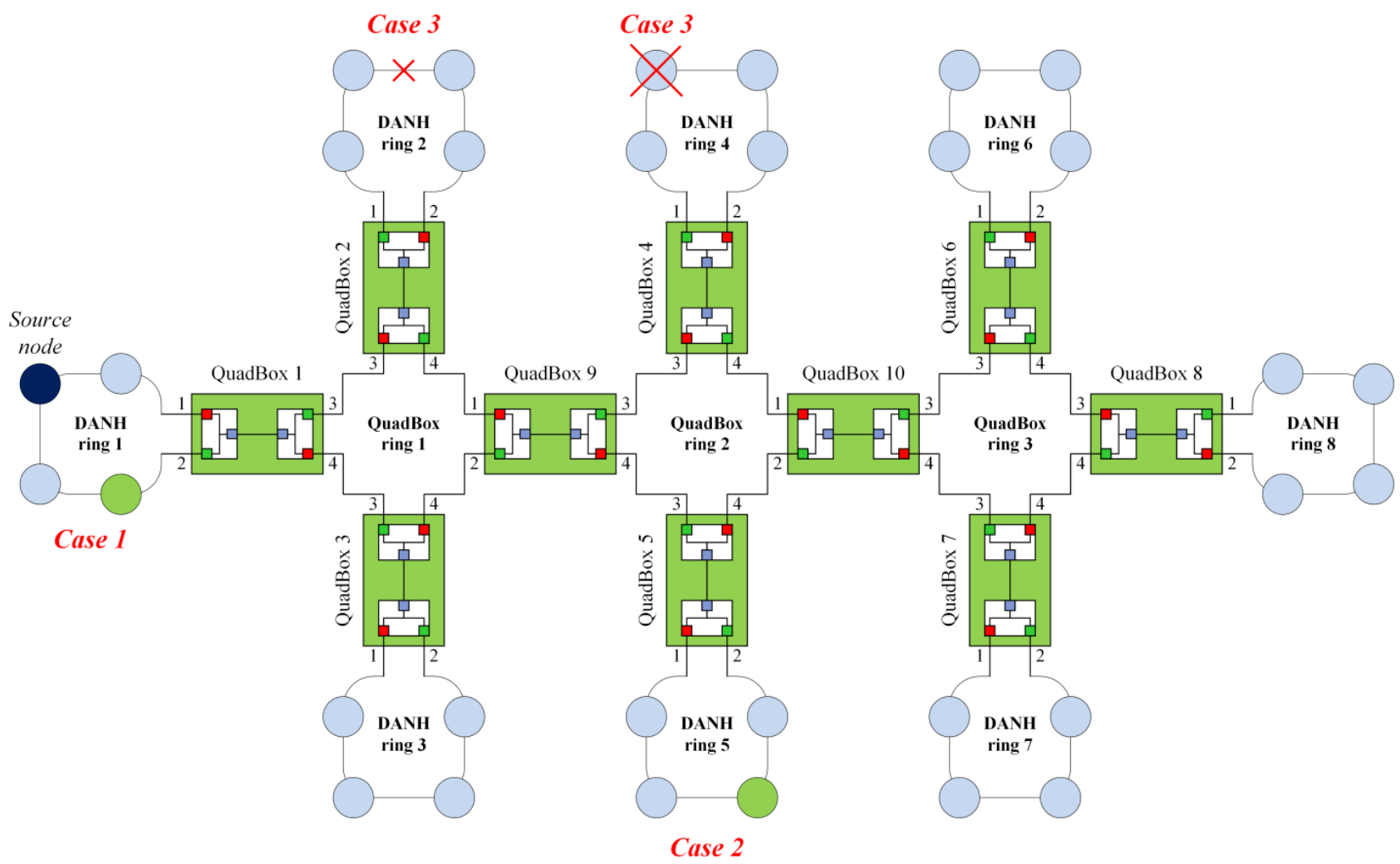

Figure 10. A sample high-availability seamless redundancy (HSR) connected-ring network.

In this paper, we consider the following cases to analyze and evaluate traffic performance:

- $\quad$ Failure-free cases: the network has no failures

- Case 1: Source and destination nodes are both in DANH ring 1

- Case 2: Source and destination nodes are in different DANH rings. The destination node is in DANH ring 5.

- Failure case: the network has a link failure and a node failure

○ Case 3: The destination node is in DANH ring 5. The network has a link failure that occurred at DANH ring 2 and a node failure at DANH ring 4.

\subsection{Notations}

In this paper, we use some parameters for network traffic performance analysis. These parameters are denoted and described in Table 1. 
Table 1. Parameters for network traffic performance analysis.

\begin{tabular}{|c|c|}
\hline Parameter & Description \\
\hline$n t_{H S R}^{1}$ & $\begin{array}{l}\text { Network traffic when a source node sends a unicast frame to a destination node under standard } \\
\text { HSR protocol }\end{array}$ \\
\hline$n t_{P L}^{1}$ & Network traffic when a source node sends a unicast frame to a destination node under the PL approach \\
\hline$n t_{P L}^{k}$ & $\begin{array}{l}\text { Network traffic when a source node sends the } k^{\text {th }} \text { unicast frame }(k>1) \text { to a destination node under } \\
\text { the PL approach }\end{array}$ \\
\hline$n t_{F H T}^{1}$ & $\begin{array}{l}\text { Network traffic when a source node sends a unicast frame to a destination node under the } \\
\text { FHT approach }\end{array}$ \\
\hline$n t_{H S R}$ & $\begin{array}{l}\text { Network traffic when a source node sends } N \text { unicast frames to a destination node under standard } \\
\text { HSR protocol }\end{array}$ \\
\hline$n t_{P L}$ & $\begin{array}{l}\text { Network traffic when a source node sends } N \text { unicast frames to a destination node under the } \\
\text { PL approach }\end{array}$ \\
\hline$n t_{F H T}$ & $\begin{array}{l}\text { Network traffic when a source node sends } N \text { unicast frames to a destination node under the } \\
\text { FHT approach }\end{array}$ \\
\hline$n t_{H S R}^{F}$ & $\begin{array}{l}\text { Network traffic in failure-case when a source node sends } N \text { unicast frames to a destination node } \\
\text { under standard HSR protocol }\end{array}$ \\
\hline$n t_{P L}^{F}$ & $\begin{array}{l}\text { Network traffic in failure-case when a source node sends } N \text { unicast frames to a destination node } \\
\text { under the PL approach }\end{array}$ \\
\hline$n t_{F H T}^{F}$ & $\begin{array}{l}\text { Network traffic in failure-case when a source node sends } N \text { unicast frames to a destination node } \\
\text { under the FHT approach }\end{array}$ \\
\hline$N R$ & A set of all rings in the network \\
\hline$N R^{-F}$ & A set of all network rings without failures \\
\hline$D R$ & A set of all DANH rings \\
\hline$D R^{-D}$ & A set of all DANH rings except the destination DANH ring \\
\hline$D R^{-F}$ & A set of DANH rings without failures \\
\hline$Q R$ & A set of all QuadBox rings \\
\hline$Q R^{-F}$ & A set of all QuadBox rings without failures \\
\hline$I R$ & A set of intermediate rings that connect the source and destination DANH ring \\
\hline$L F R$ & A set of all link-failure rings \\
\hline$N F R$ & A set of all node-failure rings \\
\hline$n_{S}$ & Total number of nodes in the source DANH ring \\
\hline$n_{D}$ & Total number of nodes in the destination DANH ring \\
\hline$n_{i}$ & Total number of nodes in the $i^{\text {th }}$ ring \\
\hline$N$ & Total number of sent frames \\
\hline
\end{tabular}

\subsection{Network Traffic Under the Standard HSR Protocol}

\subsubsection{Failure-Free Cases}

In Case 1 and Case 2, the network is failure-free. In failure-free cases, when a source node sends a unicast frame to a destination node, standard HSR protocol forwards and circulates the frame in all rings, except the destination DANH ring; two copies of the frame are delivered on each link, one in each direction. The destination DANH ring does not circulate and duplicate the frame since the destination node does not forward the frame. Therefore, the network unicast traffic in Case 1 and Case 2 is the same. Figure 11 shows the process of forwarding a unicast frame sent in Case 2 under standard HSR protocol. 
Clearly, if a frame is circulated and duplicated in a ring, the number of copies of the frame delivered in the ring is equal to twice the total number of nodes in the ring. Otherwise, the number of frame copies is equal to the total number of nodes in the ring.

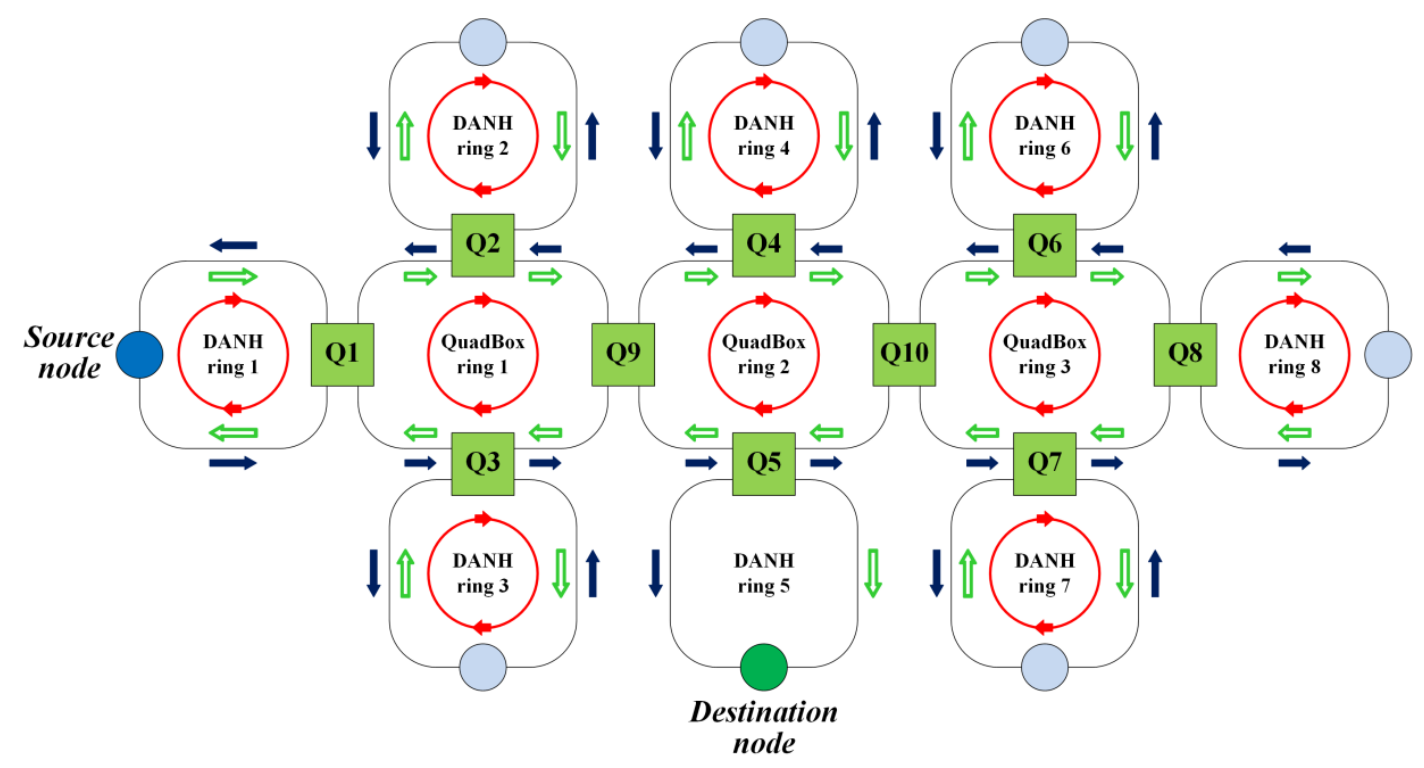

Figure 11. Network traffic under standard HSR protocol in a failure-free case.

Network traffic when the source node sends a unicast frame to the destination node in a failure-free case under standard HSR protocol, denoted by $n t_{H S R}^{1}$, is calculated as follows:

$$
n t_{H S R}^{1}=n_{D}+\sum_{i \in D R^{-D}} 2 n_{i}+\sum_{i \in Q R} 2 n_{i}
$$

where $n_{D}$ is the number of nodes in the destination DANH ring, $n_{i}$ is the number of nodes in the $i^{\text {th }}$ ring, $D R^{-D}$ is a set of all DANH rings except the destination DANH ring, and $Q R$ a set of all QuadBox rings:

$$
\sum_{i \in D R^{-D}} 2 n_{i}=\sum_{i \in D R} 2 n_{i}-2 n_{D}
$$

where $D R$ is a set of all DANH rings:

$$
\sum_{i \in D R} 2 n_{i}+\sum_{i \in Q R} 2 n_{i}=\sum_{i \in N R} 2 n_{i}
$$

where $N R$ is a set of all rings in the network.

Equation (1) can be re-written as follows:

$$
n t_{H S R}^{1}=\sum_{i \in N R} 2 n_{i}-n_{D}
$$

For the sample network in Figure 10, network traffic $n t_{H S R}^{1}$ is calculated as follows:

$$
n t_{H S R}^{1}=\sum_{i=1}^{3} 2 \times 4+\sum_{i=1}^{8} 2 \times 5-5=99 \text { (frames) }
$$


The following equation can be used in a general sense to determine network traffic under standard HSR protocol, denoted by $n t_{H S R}$, when a source node sends $N$ unicast frames to a destination node:

$$
n t_{H S R}=N\left(\sum_{i \in N R} 2 n_{i}-n_{D}\right)
$$

For the sample network in Figure 10, $n t_{H S R}=99 \mathrm{~N}$ (frames).

\subsubsection{Failure Case (Case 3)}

If a ring has a link or node failure, a unicast frame is forwarded to the ring but is not circulated and duplicated. Network traffic in the ring with a link failure, denoted by $n t^{L F}$, is determined as follows:

$$
n t^{L F}=n^{F}-1
$$

where $n^{F}$ is the number of nodes in the ring with failure.

Similarly, network traffic in a ring with a node failure, denoted by $n t^{N F}$, is determined as follows:

$$
n t^{N F}=n^{F}-2
$$

Network traffic in the failure case under standard HSR protocol when a source node sends a unicast frame to a destination node, denoted by $n t_{H S R}^{1, F}$, is calculated as follows:

$$
n t_{H S R}^{1, F}=\sum_{i \in N R^{-F}} 2 n_{i}+\sum_{i \in L F R}\left(n_{i}-1\right)+\sum_{i \in N F R}\left(n_{i}-2\right)-n_{D}
$$

where $N R^{-F}$ is a set of all network rings without failures, $L F R$ is a set of all link-failure rings, and $N F R$ is a set of all node-failure rings.

The generalized equation for calculating network unicast traffic under standard HSR protocol in failure cases when a source node sends $N$ unicast frames to a destination node is as follows:

$$
n t_{H S R}^{F}=N\left(\sum_{i \in N R^{-F}} 2 n_{i}+\sum_{i \in L F R}\left(n_{i}-1\right)+\sum_{i \in N F R}\left(n_{i}-2\right)-n_{D}\right)
$$

For the sample network in Figure 10, $n t_{H S R}^{F}=86 \mathrm{~N}$ (frames).

\subsection{Network Traffic Under PL}

\subsubsection{Failure-Free Cases}

a. Case 1:

In Case 1, we consider a situation in which source and destination nodes are both in DANH ring 1.

For the first unicast frame, PL works at the learning stage and its process of forwarding the frame is similar to that of standard HSR protocol. Network traffic under PL when the source node sends the first unicast frame to the destination node, denoted by $n t_{P L}^{1}$, is calculated using Equation (10):

$$
n t_{P L}^{1}=\sum_{i \in N R} 2 n_{i}-n_{D}
$$


For the second unicast frame, PL works at the working stage, where it locks and prunes all DANH rings except the destination DANH ring, as shown in Figure 12. However, the unicast frames are still forwarded and circulated in the source DANH ring and all QuadBox rings.

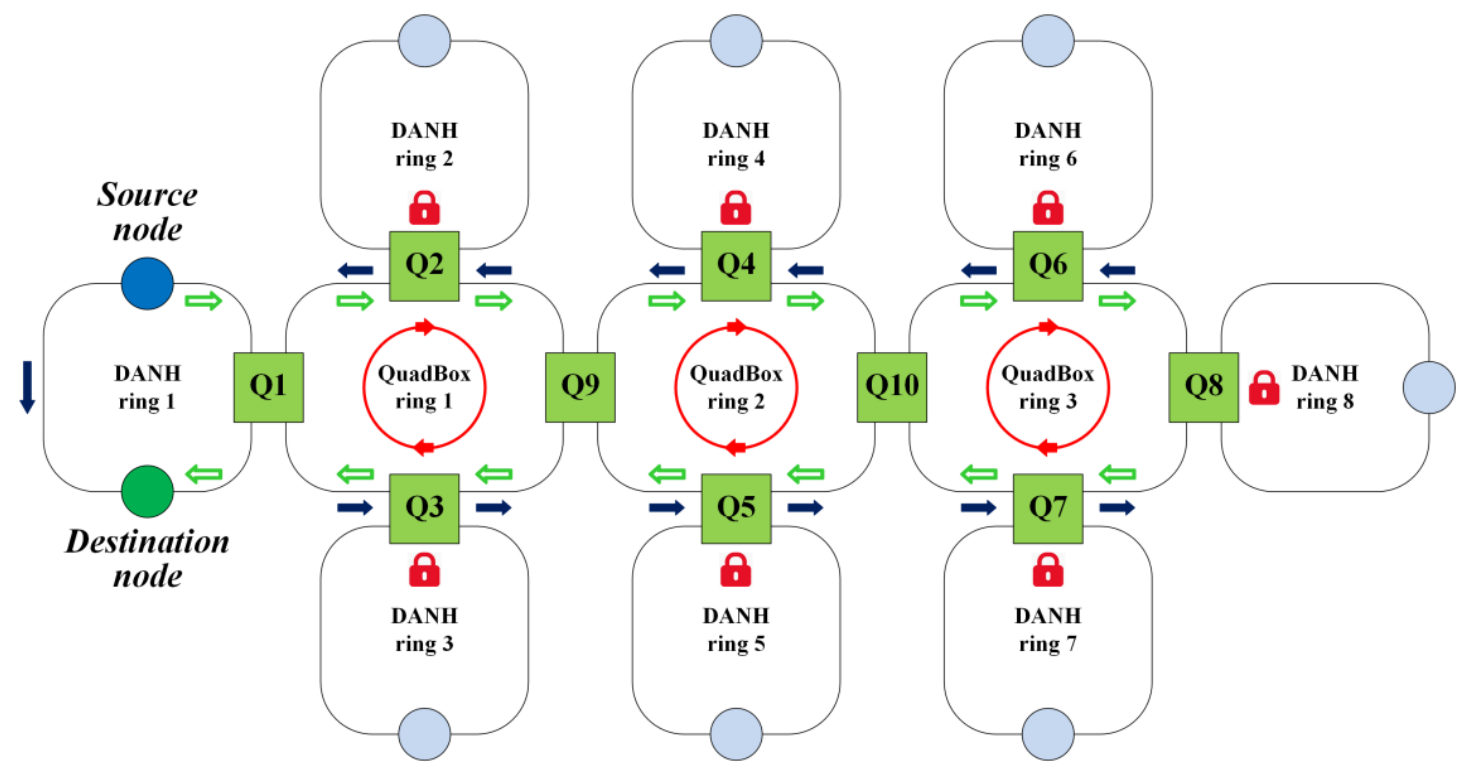

Figure 12. Network traffic under the port locking (PL) approach in Case 1.

Network traffic when the source node sends the $k^{\text {th }}$ unicast frame $(k>1)$ to the destination node, denoted by $n t_{P L}^{k}$, is determined using Equation (11):

$$
n t_{P L}^{k}=\sum_{i \in Q R} 2 n_{i}+n_{D}
$$

The generalized equation for calculating network unicast traffic under the PL approach when a source node sends $N$ unicast frames to a destination node located within the same ring is:

$$
n t_{P L}=\sum_{i \in N R} 2 n_{i}+(N-1)\left(\sum_{i \in Q R} 2 n_{i}\right)+(N-2) n_{D}
$$

For the sample network in Figure 10, $n t_{P L}=70+29 \mathrm{~N}$ (frames).

b. Case 2:

In this case, we consider a situation in which a source node in DANH ring 1 sends unicast frames to a destination node in DANH 5. For the first unicast traffic frame, network traffic is determined in Equation (10). At the working stage, PL locks and prunes all DANH rings except the source and destination rings. Unicast frames are forwarded and circulated in the source DANH ring and all QuadBox rings as shown in Figure 13. 


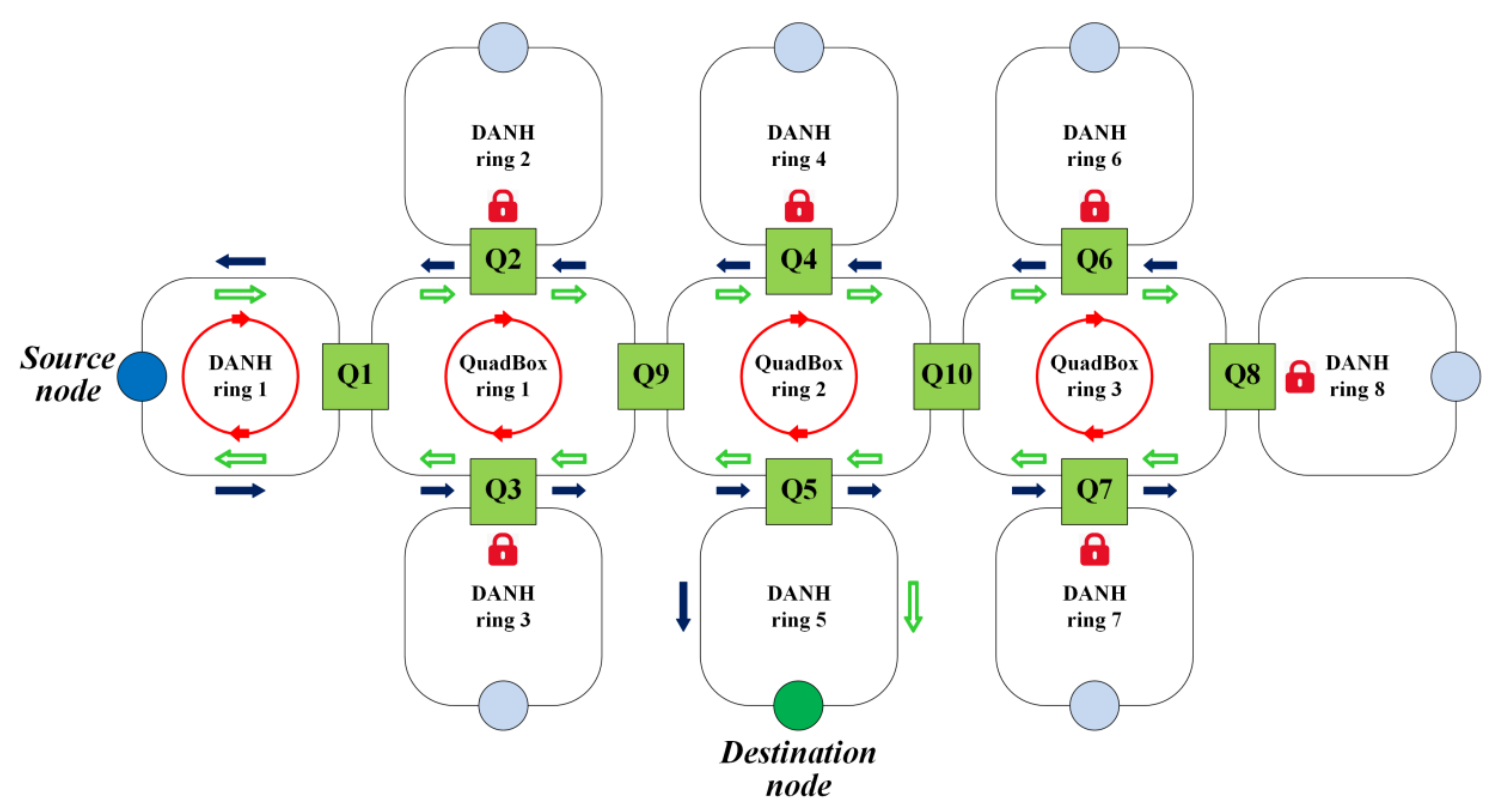

Figure 13. Network traffic under the PL approach in Case 2.

Network traffic when the source node sends the $k^{\text {th }}$ unicast frame $(k>1)$ to the destination node under the PL approach, denoted by $n t_{P L}^{k}$, is determined by Equation (13):

$$
n t_{P L}^{k}=2 n_{S}+\sum_{i \in Q R} 2 n_{i}+n_{D}
$$

where $n_{S}$ is the number of nodes in the source DANH ring.

The generalized equation for calculating network unicast traffic under the PL approach when a source node sends $N$ unicast frames to a destination node is:

$$
n t_{P L}=\sum_{i \in N R} 2 n_{i}+(N-1)\left(2 n_{S}+\sum_{i \in Q R} 2 n_{i}\right)+(N-2) n_{D}
$$

For the sample network in Figure 10, $n t_{P L}=60+39 \mathrm{~N}$ (frames).

\subsubsection{Failure Case (Case 3)}

At the learning stage, QuadBox nodes forward the first unicast traffic frame received from their QuadBox ring to their DANH ring. If a QuadBox node does not receive the frame forwarded by itself from its DANH ring, this means that its DANH ring contains the destination node of the frame, and the QuadBox continues forwarding the frames to its DANH ring at the working stage. Otherwise, it locks its ports connecting to the DANH ring at the working stage.

According to the filtering rule, PL forwards unicast frames to rings that have a link-failure or a node-failure such as DANH rings 2 and 4, regardless of the lack of a destination node in the rings as shown in Figure 14. 


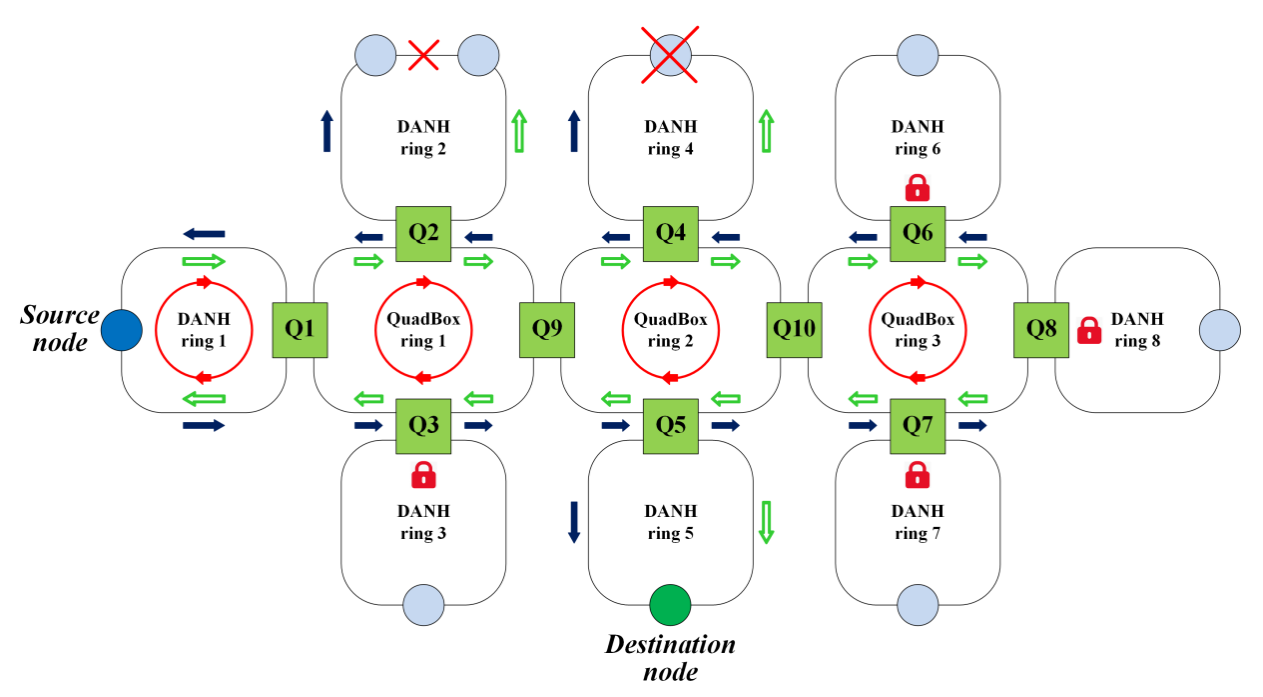

Figure 14. Network traffic under the PL approach in the failure case.

Network traffic for the first unicast frame under the PL approach in the failure case, denoted by $n t_{P L}^{1, F}$, is the same as under standard HSR protocol and is calculated as in Equation (8):

$$
n t_{P L}^{1, F}=\sum_{i \in N R^{-F}} 2 n_{i}+\sum_{i \in L F R}\left(n_{i}-1\right)+\sum_{i \in N F R}\left(n_{i}-2\right)-n_{D}
$$

Network traffic under the PL approach when the source node sends the $k^{\text {th }}$ unicast frame $(\mathrm{k}>1)$ to the destination node in the failure case, denoted by $n t_{P L}^{k, F}$, is determined by Equation (16):

$$
n t_{P L}^{k, F}=2 n_{S}+\sum_{j \in Q R^{-F}} 2 n_{j}+\sum_{i \in L F R}\left(n_{i}-1\right)+\sum_{i \in N F R}\left(n_{i}-2\right)+n_{D}
$$

where $Q R^{-F}$ is a set of all QuadBox rings without failures.

Network unicast traffic under PL in the failure case when the source node sends $N$ unicast frames to the destination node, denoted by $n t_{P L}^{F}$, is calculated as follows:

$n t_{P L}^{F}=\sum_{i \in N R^{-F}} 2 n_{i}+(N-1)\left(2 n_{S}+\sum_{j \in Q R^{-F}} 2 n_{j}\right)+N\left(\sum_{i \in L F R}\left(n_{i}-1\right)+\sum_{i \in N F R}\left(n_{i}-2\right)\right)+(N-2) n_{D}$

For the sample network in Figure 10, $n t_{P L}^{F}=40+46 \mathrm{~N}$ (frames).

Clearly, the amount of network traffic under the PL approach in the failure case is greater than in the failure-free case.

\subsection{Network Traffic Under FHT}

\subsubsection{Failure-Free Case}

a. Case 1:

When a source node sends a unicast frame to a destination node in the same DANH ring 1 under the FHT approach, QuadBox 1 filters the unicast frame and does not send it to QuadBox ring 1, as shown in Figure 15. 


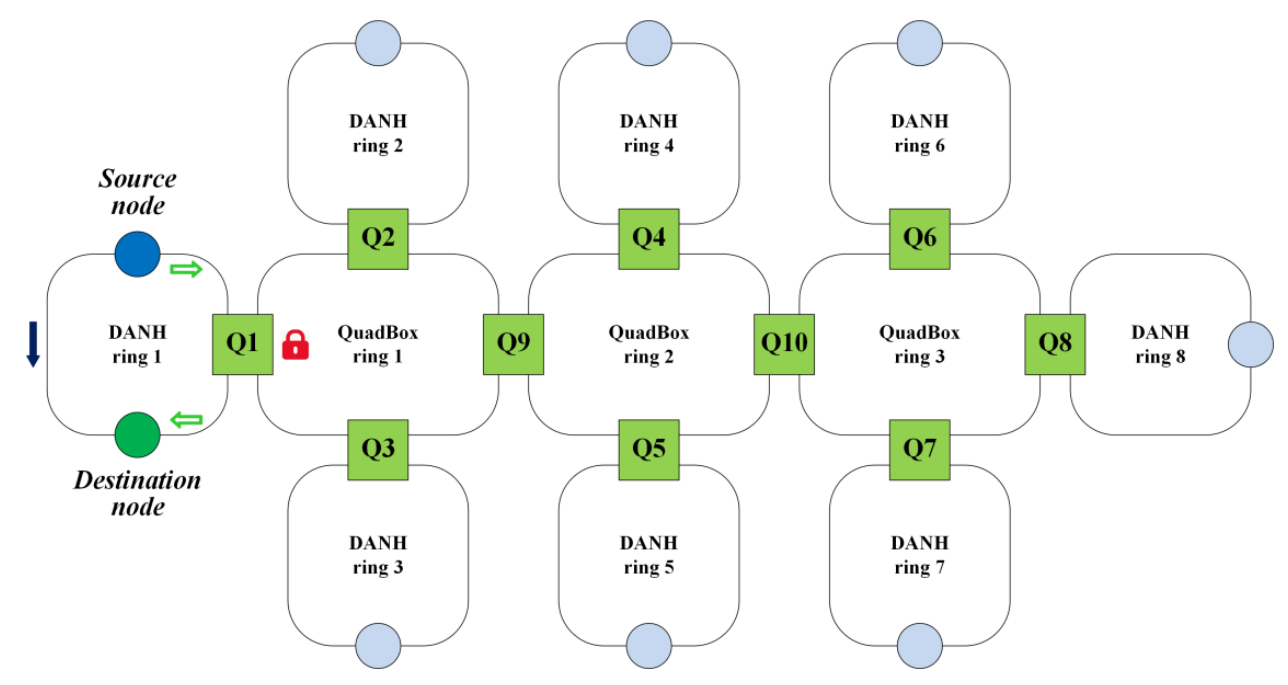

Figure 15. Network traffic under the FHT approach in Case 1.

Network traffic under FHT when the source node sends a unicast frame to the destination node in the same DANH ring, denoted by $n t_{F H T}^{1}$, is calculated using Equation (18):

$$
n t_{F H T}^{1}=n_{D}
$$

The generalized equation for calculating network unicast traffic under the FHT approach when a source node sends $N$ unicast frames to a destination node located in the same ring is:

$$
n t_{F H T}=N n_{D}
$$

For the sample network in Figure $10, n t_{F H T}=5 N$ (frames). In Case 1, FHT reduced network traffic by up to $95 \%$ compared with standard HSR and by about $84 \%$ compared with the PL approach.

\section{b. Case 2:}

In this case, when a source node sends a unicast frame to a destination node, FHT filters unicast traffic for DANH and QuadBox rings as shown in Figure 16. QuadBox rings 1 and 2 are intermediate rings connecting the source and destination DANH rings. Therefore, only QuadBox rings 1 and 2 are allowed to forward the unicast frame. However, unlike PL, which circulates and duplicates unicast frames in QuadBox rings, FHT eliminates circulation and duplication according to the traffic-forwarding rule.

Network traffic under FHT when the source node sends a unicast frame to the destination node is calculated using Equation (20):

$$
n t_{F H T}^{1}=n_{S}+n_{D}+\sum_{j \in I R} n_{j}
$$

where $I R$ is a set of intermediate rings that connect the source and destination DANH rings.

For the example network in Figure 10, IR consists of QuadBox rings 1 and 2 and $n t_{F H T}^{1}=18$.

The generalized equation for calculating network unicast traffic under the FHT approach in failure-free cases when a source node sends $N$ unicast frames to a destination node is as follows:

$$
n t_{F H T}=N\left(n_{s}+n_{d}+\sum_{j \in I R} n_{j}\right)
$$




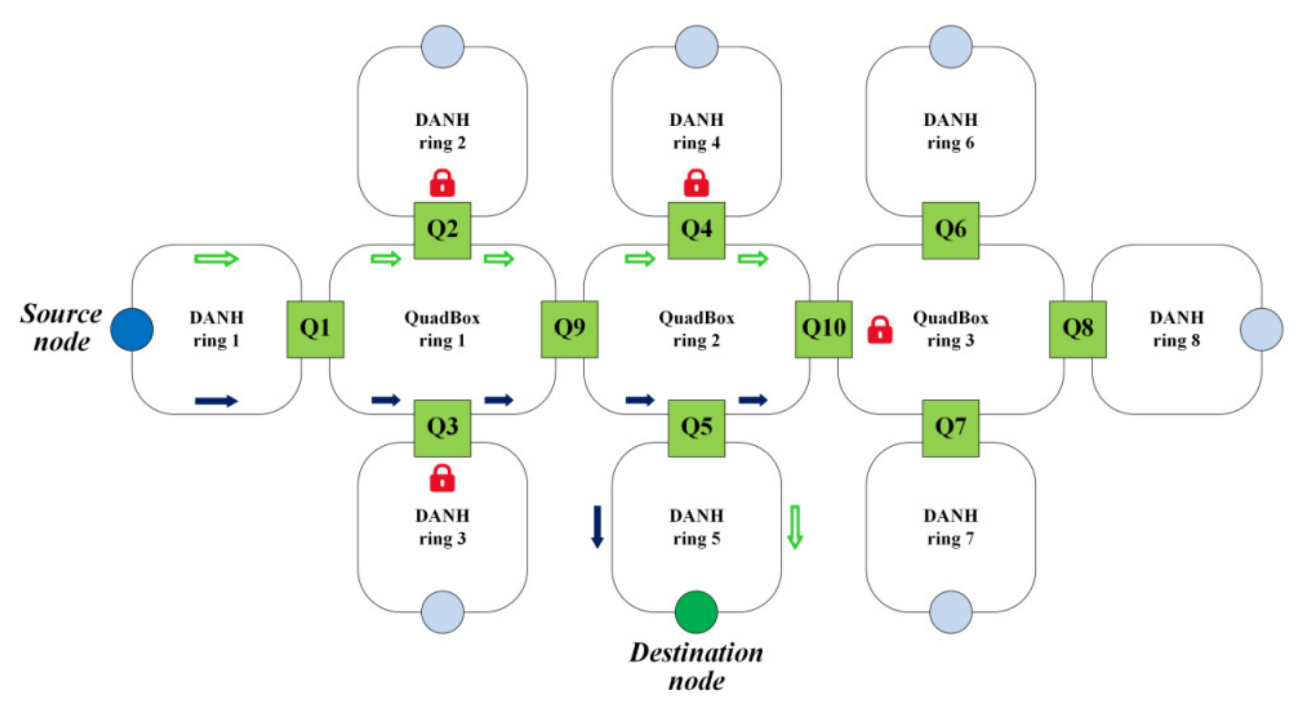

Figure 16. Network traffic under the FHT approach in Case 2.

For the sample network in Figure $10, n t_{F H T}=18 \mathrm{~N}$ (frames). In Case 2, FHT reduced network traffic by up to $82 \%$ compared with standard HSR and by about $56 \%$ compared with the PL approach.

\subsubsection{Failure Case (Case 3)}

The FHT approach filters and blocks unicast traffic for rings, except the source and destination DANH rings and the intermediate rings that connect them. In this failure case, the unicast frames are not forwarded to the failed rings, including DANH ring 2 and DANH ring 4, because the FHT approach filters the unicast traffic for the rings.

Therefore, network traffic under FHT in the failure case is the same as that in the failure-free case.

\section{Simulations}

To validate the analytical results in Section 4, various simulations were carried out using the $\mathrm{OMNeT}++$ simulation tool [12].

\subsection{Simulation Description}

We conducted three simulation experiments to validate our FHT approach. The first experiment was performed to compare the network traffic performance of FHT to that of both standard HSR protocol and the PL approach. The second experiment was implemented to validate and evaluate network traffic performance in failure cases. The third experiment was carried out to demonstrate that FHT provides much better traffic performance when the network size increases.

\subsubsection{First Experiment}

The objective of the simulation experiment was to compare the traffic performance of FHT to that of standard HSR protocol and the PL approach. We considered the sample connected-ring network consisting of eight DANH rings and three QuadBox rings, as shown in Figure 10. Each DANH ring included four DANH nodes and one QuadBox node, while each QuadBox ring consisted of four 
QuadBox nodes. Simulations were conducted for the two first cases described and analyzed in Section 4. In these simulations, a source node sent $N$ unicast traffic frames to a destination node $(N=10,20, \ldots$, 100). Network unicast traffic under HSR, PL, and FHT was recorded for comparison with the analyzed network traffic of the approaches described in Section 4.

\subsubsection{Second Experiment}

Simulations were conducted for Case 3, as described and analyzed in Section 4. In these simulations, a source node in DANH ring 1 sent $N$ unicast frames to a destination node in DANH ring $5(N=10,20, \ldots$, 100). The network had a link-failure in DANH ring 2 and a node-failure in DANH ring 4. Network unicast traffic in the failure case under the HSR, PL, and FHT approaches was recorded for comparison with the network traffic of these approaches in the failure-free case.

\subsubsection{Third Experiment}

Simulations were implemented in various connected-ring networks with different numbers of rings under standard HSR protocol and the FHT approach. The percentage of network traffic reduction for FHT compared with standard HSR was recorded from each simulation to evaluate the network traffic performance of FHT.

\subsection{Simulation Results}

Table 2 shows network unicast traffic recorded from simulations in failure-free cases and failure cases under standard HSR protocol, the PL approach, and the FHT approach.

Table 2. Network traffic frames in three simulated cases.

\begin{tabular}{|c|c|c|c|c|c|c|c|c|c|}
\hline \multirow{4}{*}{ Number of sent frames } & \multicolumn{9}{|c|}{ Total number of network traffic frames } \\
\hline & \multicolumn{6}{|c|}{ Failure-free cases } & \multirow{2}{*}{\multicolumn{3}{|c|}{$\begin{array}{c}\text { Failure case } \\
\text { Case } 3\end{array}$}} \\
\hline & \multicolumn{3}{|c|}{ Case 1} & \multicolumn{3}{|c|}{ Case 2} & & & \\
\hline & HSR & PL & FHT & HSR & PL & FHT & HSR & PL & FHT \\
\hline 10 & 1,000 & 370 & 50 & 990 & 450 & 200 & 860 & 500 & 200 \\
\hline 20 & 2,000 & 670 & 100 & 1,980 & 840 & 400 & 1,720 & 960 & 400 \\
\hline 30 & 3,000 & 970 & 150 & 2,970 & 1,230 & 600 & 2,580 & 1,420 & 600 \\
\hline 40 & 4,000 & 1,270 & 200 & 3,960 & 1,620 & 800 & 3,440 & 1,880 & 800 \\
\hline 50 & 5,000 & 1,570 & 250 & 4,950 & 2,010 & 1,000 & 4,300 & 2,340 & 1,000 \\
\hline 60 & 6,000 & 1,870 & 300 & 5,940 & 2,400 & 1,200 & 5,160 & 2,800 & 1,200 \\
\hline 70 & 7,000 & 2,170 & 350 & 6,930 & 2,790 & 1,400 & 6,020 & 3,260 & 1,400 \\
\hline 80 & 8,000 & 2,470 & 400 & 7,920 & 3,180 & 1,600 & 6,880 & 3,720 & 1,600 \\
\hline 90 & 9,000 & 2,770 & 450 & 8,910 & 3,570 & 1,800 & 7,740 & 4,180 & 1,800 \\
\hline 100 & 10,000 & 3,070 & 500 & 9,900 & 3,960 & 2,000 & 8,600 & 4,640 & 2,000 \\
\hline
\end{tabular}

Figure $17 \mathrm{a}$ shows the comparison of analytical traffic performance and Figure $17 \mathrm{~b}$ shows the comparison of simulated traffic performance for FHT compared with standard HSR protocol and the PL approach in Case 1. In this case, the analytical and simulation results show that FHT reduces network traffic by up to $95 \%$ compared with standard HSR and by about $84 \%$ compared with the PL approach. 
Figure 18a shows the comparison of analytical traffic performance and Figure $18 \mathrm{~b}$ shows the comparison of simulated traffic performance for FHT compared with standard HSR protocol and the PL approach in Case 2. In this case, the analytical and simulation results show that FHT reduces network traffic by up to $82 \%$ compared with standard HSR and by about $56 \%$ compared with the PL approach.

Figure 19a shows the comparison of network traffic performance in the failure case to that in the failure-free case, and Figure 19b shows the percentage of network traffic reduction under FHT when network size increases.

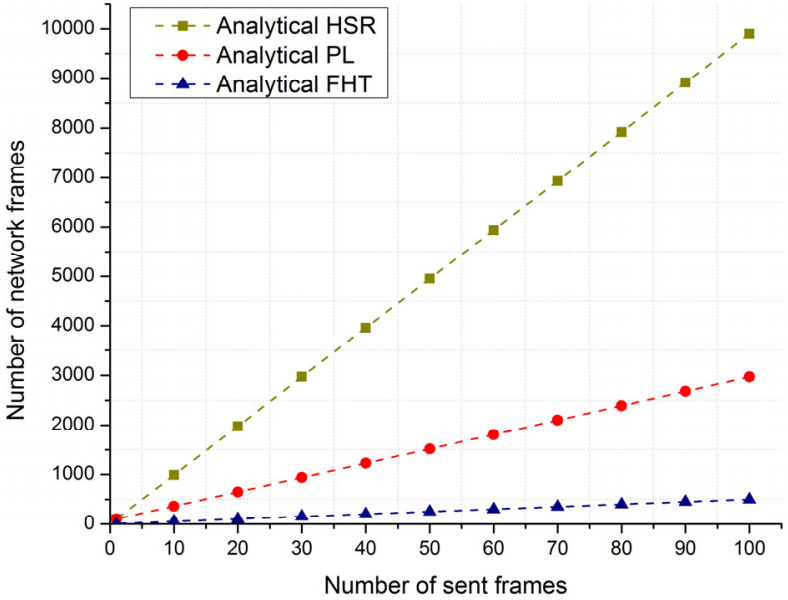

(a) Analytical traffic performance

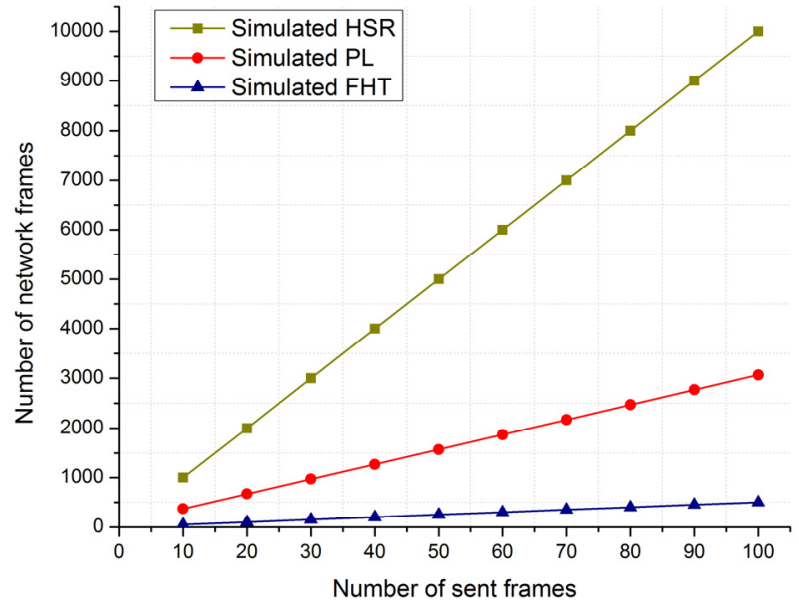

(b) Simulated traffic performance

Figure 17. Comparison of analytical and simulated traffic performance between HSR, PL, and FHT in Case 1.

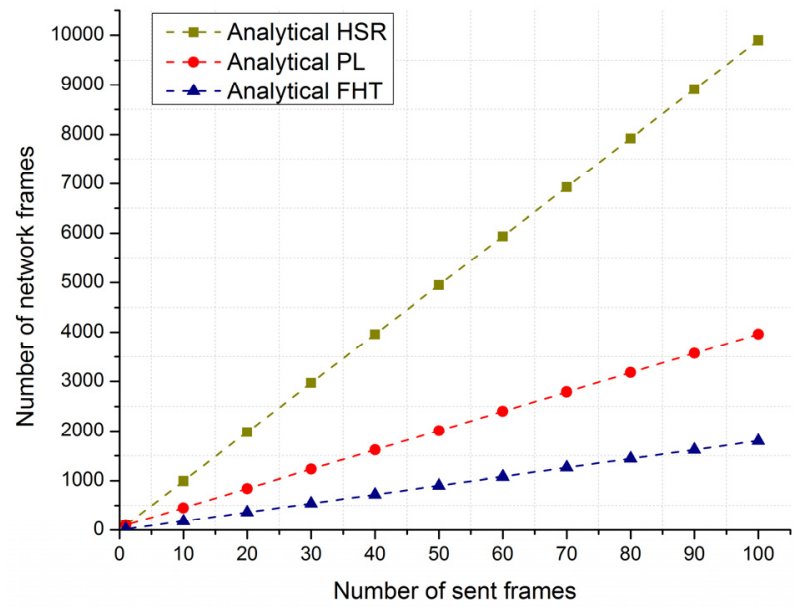

(a) Analytical traffic performance

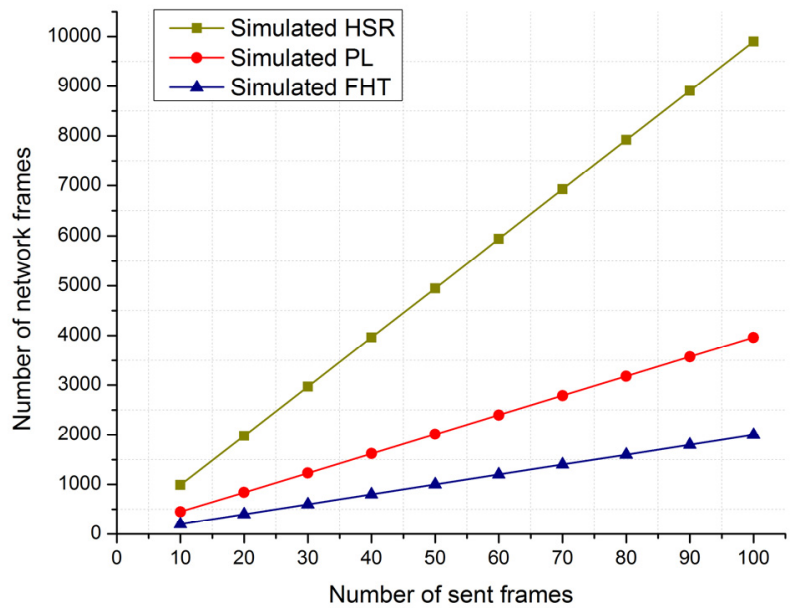

(b) Simulated traffic performance

Figure 18. Comparison of analytical and simulated traffic performance between HSR, PL, and FHT in Case 2. 


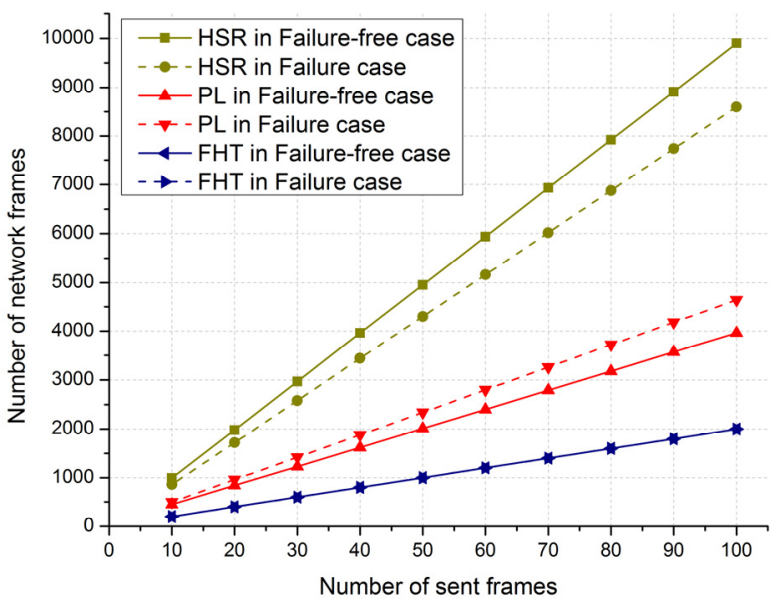

(a) The second experiment

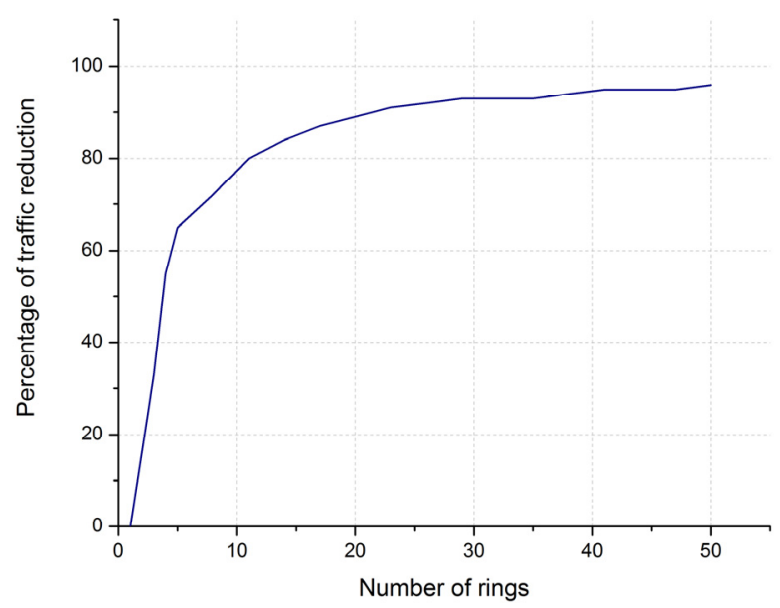

(b) The third experiment

Figure 19. Simulation results of the second experiment and the third experiment.

\subsection{Discussion}

The simulation results demonstrate that the traffic performance of the proposed FHT is much better than standard HSR protocol and the PL approach. Unlike PL, which only prunes unicast traffic for DANH rings, the FHT approach filters unicast traffic for DANH rings as well as QuadBox rings. In addition, FHT removes circulated unicast traffic in rings. Therefore, FHT significantly reduces HSR unicast network traffic compared with standard HSR protocol and PL. Numerically, FHT reduces unicast network traffic by $82 \%$ compared with standard HSR protocol and by $56 \%$ compared with PL. Of particular importance, FHT reduces network traffic by up to $95 \%$ compared with standard HSR protocol and by $84 \%$ compared with PL when the source node and destination node are connected to the same DANH ring.

In the case of link-failures or node-failures in DANH rings, network unicast traffic is increased under the PL approach and is not changed under FHT. Additionally, unlike PL, which cannot work with connected-ring networks that have an intermediate DANH ring connected to two other rings, FHT works with any connected-ring networks.

The simulation results also show that FHT provides a much better percentage of network traffic reduction when the network size increases. This is because FHT allows network traffic to be forwarded in the source and destination DANH rings and only intermediate rings that connect the source and destination DANH rings. Table 3 shows a summary of the traffic-filtering features of standard HSR protocol, PL and FHT.

Table 3. Summary of traffic-filtering features.

\begin{tabular}{cccc}
\hline Traffic-Filtering Features & Standard HSR & PL & FHT \\
\hline Traffic-filtering for DANH rings & No & Yes & Yes \\
\hline Traffic-filtering for QuadBox rings & No & No & Yes \\
\hline Removing circulated traffic & No & No & Yes \\
\hline
\end{tabular}




\section{Conclusions}

In this paper, we proposed a novel approach called FHT, which filters HSR network unicast traffic in connected-ring networks. The FHT approach filters unicast traffic for both DANH rings and QuadBox rings. FHT also prevents networks from circulating unicast traffic in the rings. Therefore, FHT significantly reduces unicast traffic compared with standard HSR protocol and the PL approach. Analytical and simulation results showed that, for our sample network, FHT reduced network unicast traffic by about $82 \%$ compared with the standard HSR protocol and by $56 \%$ compared with the PL approach. Perhaps most importantly, FHT reduces network unicast traffic by up to $95 \%$ compared with standard HSR protocol and by $84 \%$ compared with the PL approach when source and destination nodes are connected to the same DANH ring. In addition, the network traffic performance of FHT is most improved when the network size increases. FHT overcomes the limitations of the PL approach because it can work with all connected-ring topologies and filter unicast traffic for rings that have any link-failure or node-failure. FHT is an efficient approach to significantly reduce unicast traffic in HSR networks. It can be implemented for HSR nodes in time-critical and mission-critical systems such as SAS.

Our future work will involve developing and applying the FHT approach for all network traffic types: unicast as well as multi-broadcast.

\section{Acknowledgments}

This work was supported by the 2015 MPEES Advanced Research Center Fund of Myongii University.

\section{Author Contributions}

Nguyen Xuan Tien and Jong Myung Rhee conceived and developed the ideas behind the research. Nguyen Xuan Tien carried out the performance analysis and simulations, and wrote the paper under supervision of Jong Myung Rhee. Jong Myung Rhee supervised the research and finalized the paper.

\section{Conflicts of Interest}

The authors declare no conflict of interest.

\section{References}

1. Nsaif, S.A.; Rhee, J.-M. DVP: A Novel High-Availability Seamless Redundancy (HSR) Protocol Traffic-Reduction Algorithm for a Substation Automation System Network. Energies 2014, 7 , 1792-1810.

2. IEC Standard. High-Availability Automation Networks, Part 2: Media Redundancy Protocol (MRP); IEC 62439-2; The International Electrotechnical Commission: Geneva, Switzerland, 2010.

3. IEC Standard. High-Availability Automation Networks, Part 4: Cross-Network Redundancy Protocol (CRP); IEC 62439-4; The International Electrotechnical Commission: Geneva, Switzerland, 2010.

4. IEC Standard. High-Availability Automation Networks, Part 5: Beacon Redundancy Protocol (BRP); IEC 62439-5; The International Electrotechnical Commission: Geneva, Switzerland, 2010. 
5. IEC Standard. High-Availability Automation Networks, Part 3: Parallel Redundancy Protocol (PRP) and High-Availability Seamless Redundancy (HSR); IEC 62439-3; The International Electrotechnical Commission: Geneva, Switzerland, 2012.

6. IEEE 801.1aq Standard-Shortest Path Bridging (SPB). Available online: http://www.ieee802.org/ 1/pages/802.1aq.html (accessed on 2 June 2015).

7. Kirrmann, H.; Kleineberg, O. Seamless and low-cost redundancy for Substation Automation Systems (High availability Seamless Redundancy, HSR). In Proceedings of the Power and Energy Society General Meeting, San Diego, CA, USA, 24-29 July 2011.

8. Nsaif, S.A.; Rhee, J.M. Improvement of high-availability seamless redundancy HSR traffic performance for smart grid communications. J. Commun. Netw. 2012, 14, 653-661.

9. Tien, N.X.; Nsaif, S.A.; Rhee, J.M. High-Availability Seamless Redundancy (HSR) Traffic Reduction Using Optimal Dual Paths (ODP). In Proceedings of the International Conference on Green and Human Information Technology (ICGHIT), DaNang, Vietnam, 4-6 February 2015.

10. Hong, S.; Joe, I. A Novel Packet Transmission Scheme with Different Periods According to the HSR Ring Direction in Smart Grid. In Proceedings of the 4th International Conference on Future Generation Information Technology, Kangwondo, Korea, 16-19 December 2012.

11. Abdulsalam, I.R.; Rhee, J.M. Improvement of High-Availability Seamless Redundancy (HSR) Unicast Traffic Performance Using Port Locking. In Proceedings of the Fourth World Congress on Software Engineering, Hong Kong, China, 3-4 December 2013.

12. OMNeT++ Version 4.6 Simulator. Available online: http://www.omnetpp.org/ (accessed on 15 January 2015).

(C) 2015 by the authors; licensee MDPI, Basel, Switzerland. This article is an open access article distributed under the terms and conditions of the Creative Commons Attribution license (http://creativecommons.org/licenses/by/4.0/). 